\title{
Effects of insulin on the reversal of myocardial contractility after bupivacaine-induced cardiac asystole in vitro
}

Hyun Joo Kim

Yonsei University College of Medicine

Ja Rang Jung

Yonsei University College of Medicine

Carl Lynch III

University of Virginia Health System

Wyun Kon Park ( $\nabla$ wkp7ark@yuhs.ac)

Yonsei University College of Medicine https://orcid.org/0000-0002-5204-3921

Research article

Keywords: Bupivacaine, Cardiac toxicity, Insulin, Myocardial contraction, Guinea pigs

Posted Date: October 14th, 2019

DOI: https://doi.org/10.21203/rs.2.11217/v3

License: (c) (i) This work is licensed under a Creative Commons Attribution 4.0 International License.

Read Full License 


\section{Abstract}

Background: Insulin-glucose treatment effectively reverses severe bupivacaine (BPV)-induced myocardial depression or cardiovascular collapse in in vivo. However, the mechanisms for the recovery are poorly defined. Methods: Using the guinea pig myocardium, cumulative concentration-responses on contractile forces for insulin or insulin combined with $33 \mathrm{mM}$ glucose (insulin/glucose) were measured. After achieving asystole by $500 \mu \mathrm{M}$ BPV, different concentrations of insulin or insulin/glucose were applied to determine the recovery of stimulated contractile responses and contractions in either recirculating or nonrecirculating (washout) condition. Because we did not observe any recovery from asystole with insulin treatment in the recirculating condition, further experiments were performed whether intermittent contractile responses (conduction block) could be reversed by insulin. In the washout condition, after achieving asystole, the muscles were washed with the Tyrode solution containing insulin or insulin/glucose for 60 minutes. After achieving asystole, BPV concentrations in the Tyrode solution in the presence or absence of insulin for 60 minutes were measured. Results: There were similar concentrationdependent decreases in contractility in both the insulin and insulin/glucose groups. Neither insulin nor insulin/glucose restored the stimulated contractile responses from conduction disturbance or asystole induced by BPV in the continued presence of BPV. In the BPV-washout condition, while superfusion with a control (plain Tyrode) solution for 60 minutes after achieving asystole by BPV restored contractility to approximately $60 \%$ of the baseline, time-dependent complete recovery was observed in the insulin- and insulin/glucose-treated groups. At each time period from asystole to 60 minutes, the BPV concentrations in the insulin-treated group were slightly lower than those in the control group. Conclusions: Neither insulin nor insulin/glucose treatment does not rescue $\mathrm{Na}+$ channel function to reverse BPV-induced cardiac conduction block or asystole regardless of improved cardiac performance, possibly due to improved myocardial energetics in isolated in vitro animal myocardium model. These findings suggest that treatment with insulin or insulin/glucose is desirable for metabolic energy supply to the myocardium in cases of BPV-induced cardiac collapse. However, considering the importance of decreased BPV concentrations in cardiac tissues, insulin or insulin/glucose may not achieve satisfactory outcomes.

\section{Background}

Bupivacaine (BPV) impairs oxidative metabolism of fatty acids in cardiomyocytes by inhibition of carnitine-acylcarnitine translocase [1], one of the enzymes necessary for the transport of fatty acids into mitochondria, which presumably contributes to BPV-induced cardiac toxicity. When BPV infusion impairs utilization of these substrates, an alternative fuel (e.g. carbohydrate) can be used to promote cardiac ATP synthesis [2]. An infusion of insulin will increase glucose uptake, and, through the glycolytic pathway, provide increased concentrations of pyruvate [3], resulting in replenishment of ATP and improvement in myocardial performance. In in vivo studies using dogs, insulin-glucose-potassium treatment effectively reversed severe BPV-induced myocardial depression [4] or cardiovascular collapse [5], whereas no control dogs were resuscitated. In an in vitro study using a Langendorff preparation in rats, Stehr et al. [6] also found that insulin application reversed L-BPV-induced myocardial contractile depression, but did not 
improve impaired myocardial conduction induced by L-BPV. Insulin-glucose-potassium treatment in conjunction with chest compression effectively reversed mean arterial blood pressure in BPV-induced cardiovascular collapse in dogs, however, a longer period of time was also required to reverse impaired myocardial conduction [5]. These results suggest that insulin treatment may not be effective for reversal of BPV-induced conduction disturbances, or even asystole. To restore impaired myocardial conduction, the BPV content in cardiac tissues should be sufficiently lowered to restore $\mathrm{Na}^{+}$channel function. BPV is easily taken up by the lipid micelles of a lipid emulsion due to its high lipophilicity $(\log P=3.64)$ [7], however, it may not be partitioned by insulin because the action of insulin is purely metabolic. Thus, insulin itself may not reduce BPV content in cardiac tissues, resulting in no recovery of conduction disturbances or asystole.

Therefore, we hypothesized that insulin has no effect on the reversal of BPV-induced conduction impairments or asystole regardless of improvement in BPV-induced myocardial energy deficiency and cardiac performance in isolated in vitro animal myocardium model.

\section{Methods}

To elucidate the mechanisms related to contractile recovery after BPV-induced cardiac asystole, isolated guinea pig papillary muscles were treated with insulin either in the continued presence of BPV or during its washout, and their contributions to the improvement of contractile performance were examined.

\section{Preparation of cardiac muscles}

According to a procedure approved by the Yonsei University College of Medicine Animal Research Committee, hearts were removed from 400-450 g male guinea pigs (Dunkin Hartley) after inhalation of sevoflurane (3-4 vol\%) in an enclosed cage. Guinea pigs were obtained from the Institutional Animal Care Facility (Yonsei University Health System, Seoul, Korea). The right ventricular papillary muscles were excised, mounted horizontally in a tissue bath, and superfused at $37^{\circ} \mathrm{C}$ with modified normal Tyrode solution (Tyrode solution) (118 mM NaCl, $5 \mathrm{mM} \mathrm{KCl}, 2 \mathrm{mM} \mathrm{CaCl}_{2}, 1.2 \mathrm{mM} \mathrm{MgSO}_{4}, 25 \mathrm{mM} \mathrm{NaHCO}_{3}^{-} ; 11$ $\mathrm{mM}$ glucose, and $0.10 \mathrm{mM}$ EDTA) [8]. The solution was recirculated through the bath from a reservoir containing $100 \mathrm{ml}$ of Tyrode solution through which $95 \% \mathrm{O}_{2}$ and $5 \% \mathrm{CO}_{2}$ were bubbled, maintaining a pH of $7.4 \pm 0.05$. The tendinous end of the papillary muscle was attached to a Grass FT03 force transducer (Grass Instruments, Quincy, MA, USA) while the other end of the muscle was pinned to the bottom of the tissue bath. For stabilization, muscles were field-stimulated using a Grass S44 stimulator (Grass Instruments, Quincy, MA, USA) at $0.5 \mathrm{~Hz}$ for 60 minutes. After stabilization, contractile forces were obtained at $1.2 \mathrm{~Hz}$ for 15 minutes as a baseline. The $1.2 \mathrm{~Hz}$ stimulation was continuously applied during the entire experimental period after stabilization. The excised papillary muscles were randomly allocated into 3 groups: control $(n=10)$ insulin-treated $(n=39)$, and insulin-treated in the presence of high concentration of glucose $(33 \mathrm{mM})(\mathrm{n}=31)$ groups. Forces were continuously recorded and analyzed using a Powerlab ${ }^{\circledR}$ Data Analysis System (Chart v.7.0, ADInstruments, Sydney, Australia). Contractile 
function parameters [peak force (PF) and maximum rate of force development (dF/dt-max)] were also recorded. $\mathrm{dF} / \mathrm{dt}$-max values were used as a measure of contractility.

\section{Experimental protocols}

\section{Effects of insulin on contractility}

Following baseline measurements, muscles were exposed to each drug concentration $\left(10^{-3}, 10^{-2}, 10^{-1}, 2 \mathrm{x}\right.$ $10^{-1}$, and $5 \times 10^{-1}$ units $/ \mathrm{ml}$ ) of insulin (Humulin insulin R, Eli Lilly and Company, Indianapolis, IN, USA) for 10 minutes. To further define the effect of insulin in the presence of a higher-than-baseline concentration of glucose, concentration-responses for insulin were also obtained following the 15-minute exposure to $33 \mathrm{mM}$ glucose. The insulin concentrations were determined based on the concentrations used by Armin and Bolte [9] in their study using guinea pig papillary muscles. Considering that the circulating lines were a PVC tubing system, $30 \%$ more insulin was added to each concentration to compensate for absorption of insulin to the tubing [10] in all insulin-related experiments.

\section{Recirculation experiments - continuing BPV}

To determine the insulin concentrations to use in asystole experiments, changes in contractile force in response to increasing concentrations of insulin following application of $50 \mu \mathrm{M} B P V$ were measured. After obtaining baseline measurements, $50 \mu \mathrm{M}$ BPV was applied for 15 minutes followed by sequential exposure to each concentration of insulin $\left(10^{-3}, 10^{-2}, 10^{-1}, 2 \times 10^{-1}\right.$, and $5 \times 10^{-1}$ units $\left./ \mathrm{ml}\right)$. Each concentration of insulin was maintained for 10 minutes. To further define the additive effect of glucose, muscles were exposed to $33 \mathrm{mM}$ glucose after obtaining contractile depression with $50 \mu \mathrm{M}$ BPV. Cumulative concentration-responses for insulin were obtained following a 15-minute exposure to $33 \mathrm{mM}$ glucose.

To assess recovery from BPV-induced cardiac asystole, $500 \mu \mathrm{M}$ BPV was applied after obtaining baseline measurements. If asystole did not develop for 20 minutes, $100 \mu \mathrm{M}$ BPV was increased. If asystole did not develop despite a 600- $\mu \mathrm{M}$ BPV application for 20 minutes, we stopped the experiment. Five minutes after obtaining asystole, muscles were exposed to $10^{-1}, 2 \times 10^{-1}$, and $5 \times 10^{-1}$ units $/ \mathrm{ml}$ of insulin. Each insulin concentration was maintained for 20 minutes. To further define the additive effect of glucose, muscles were exposed to $33 \mathrm{mM}$ glucose in Tyrode solution after obtaining asystole, followed by application of $10^{-1}, 2 \times 10^{-1}$, and $5 \times 10^{-1}$ units $/ \mathrm{ml}$ of insulin.

Because we did not observe any recovery from asystole in muscles treated with insulin with or without 33 $\mathrm{mM}$ glucose, we performed further experiments to confirm whether intermittent contractile responses (conduction block) could be reversed by insulin. When conduction block did not develop after a 15-minute application of $200 \mu \mathrm{M} \mathrm{BPV}$, the concentration was increased to $250 \mu \mathrm{M}$. If there was no conduction block, the BPV concentration was further increased to $300 \mu \mathrm{M}$; if conduction block did not develop after 15 minutes, the experiment was stopped. After obtaining regular intermittent contractile responses, $10^{-1}, 2 \mathrm{x}$ $10^{-1}$, and $5 \times 10^{-1}$ units $/ \mathrm{ml}$ of insulin were applied. To examine the additive effect of glucose, at 5 minute 
after obtaining regular intermittent contractile responses, muscles were exposed to $33 \mathrm{mM}$ glucose in Tyrode solution for 5 minutes, and the insulin concentrations were then increased sequentially. Each insulin concentration was maintained for 20 minutes.

\section{Measurements of BPV concentrations in solutions}

BPV concentrations in solution were measured under the recirculating condition at 3 minutes after applying $500 \mu \mathrm{M}$ BPV, 5 minutes after asystole, and after 15, 30, 45, and 60 minutes, in the control solution or the control solution containing $2 \times 10^{-1}$ unit/ml of insulin. In the control solution- or insulintreated group, one milliliter of solution was collected at each time point. The samples were stored at $-80^{\circ} \mathrm{C}$ until analysis. BPV in Tyrode solution was extracted by methylene chloride. After shaking, the organic solution was separated by centrifugation at 13,000 rpm for 3 minutes. It was dried using $\mathrm{N}_{2}$ gas and reconstituted in $5 \mathrm{mM}$ ammonium acetate:acetonitrile (40:60, v/v). A $0.5-\mu l$ aliquot of the solution was analyzed using an LS/MS/MS system. Lidocaine was used as the internal standard.

\section{Non-recirculation experiments - BPV washout}

Using the same experimental protocol as in recirculation experiments, 500-600 $\mu \mathrm{M}$ BPV was applied. Five minutes after obtaining asystole, muscles were washed out with Tyrode solution containing $2 \times 10^{-1}$ unit/ml of insulin for 60 minutes. To define the additive effect of glucose, muscles were washed with Tyrode solution containing $33 \mathrm{mM}$ glucose and $2 \times 10^{-1} \mathrm{unit} / \mathrm{ml}$ of insulin for 60 minutes. Plain Tyrode solution was used as a control.

Insulin was purchased from Eli Lilly Company (Indianapolis, IN, USA). All other chemicals were purchased from Sigma-Aldrich Chemicals Company (St. Louis, MO, USA).

\section{Statistical analysis}

Because of variations in baseline values from one muscle to another, alterations in contractile function were expressed as a percentage of baseline. Differences in the time to the appearance of asystole ( $\left.T_{\text {asystole }}\right)$, the time to the appearance of the first contractile response $\left(T_{1 s t R}\right)$, and the time to the first appearance of regular contraction $\left(T_{R R}\right)$ among treatment groups in the washout condition were analyzed using one-way analysis of variance (ANOVA) followed by Bonferroni post hoc tests. Repeated measures ANOVA was used to test for differences between baseline and time-dependent changes in contractile forces in the recirculation condition. To compare the two groups at each concentration for 60 minutes, unpaired t-test was used. Linear mixed models (LMMs) were used to compare the BPV concentrations between control preparations and those treated with insulin for 60 minutes. LMMs were also used to compare the changes in contractile forces during recovery among control, insulin-, and insulin and $33 \mathrm{mM}$ glucose-treated groups in the washout condition. In all analyses using LMMs, post hoc analysis was performed in which two-tailed $\mathrm{P}$ values were adjusted by the Bonferroni correction to control the significance level. All data are presented as the mean \pm SD. A $P$ value $<0.05$ was considered significant. The Shapiro-Wilk test was used as a test of normality test. Non-parametric tests were used when data did 
not meet the assumption of normality. No statistical power calculation was conducted prior to the study. The sample size was based on our previous experiences with this design

\section{Results}

\section{Effects of insulin on contractility}

No contractile depression was observed at $10^{-3}, 10^{-2}$, or $10^{-1}$ unit/ml of insulin, whereas $2 \times 10^{-1}$ and $5 \mathrm{x}$ $10^{-1}$ units/ml of insulin depressed contractility by approximately $20 \%$ and $30 \%$, respectively. Similar contractile depression was observed, using the Tyrode solution containing $33 \mathrm{mM}$ glucose (Fig. 1).

\section{Recirculation experiments - continuing BPV}

\section{Effects of insulin on BPV-induced contractile depression}

Fifty $\mu \mathrm{M}$ BPV depressed contractility by approximately $55 \%$. After achieving contractile depression by 50 $\mu \mathrm{M} B P V$, application of insulin from $10^{-3}$ to $5 \times 10^{-1}$ units $/ \mathrm{ml}$ did not alter the contractility (Fig. 2). In the presence of $33 \mathrm{mM}$ glucose in Tyrode solution, each concentration of insulin, excluding $2 \times 10^{-1}$ and $5 \mathrm{x}$ $10^{-1}$ units $/ \mathrm{ml}$, modestly but significantly increased contractility by approximately $10 \%$ compared to that of $50 \mu \mathrm{M}$ BPV. Insulin at $5 \times 10^{-1} \mathrm{unit} / \mathrm{ml}$ did not attenuate the BPV-induced decrease in contractility (Fig. 2).

\section{Effects of insulin on BPV-induced asystole}

In all muscles superfused with either normal or $33 \mathrm{mM}$ glucose in Tyrode solution, asystole developed approximately 7 - 10 minutes after exposure to $500 \mu \mathrm{M}$ BPV (Table 1). Administration of insulin, 10-1, $2 \mathrm{x}$ $10^{-1}$, and $5 \times 10^{-1}$ units $/ \mathrm{ml}$, in the absence (Fig. $3 \mathrm{~A}$ ) or presence of $33 \mathrm{mM}$ glucose did not recover any stimulated contractile responses during the 60 minute-experimental period.

\section{Effects of insulin on intermittent contractile responses (conduction block)}

Intermittent contractile responses appeared at approximately 11 minutes after exposure to intermediate concentrations of BPV (200-300 $\mu \mathrm{M})$. Intermediate concentration of BPV significantly decreased the stimulated contractile responses by $47 \%$ (approximately 2:1 conduction block), which was sustained following successive application of $10^{-1}, 2 \times 10^{-1}$, and $5 \times 10^{-1}$ units/ml of insulin (Fig. 3B). An intermediate concentration of BPV depressed contractility by approximately $60 \%$; this did not improve but was sustained following exposure to each concentration of insulin (Fig. 3B). Similar results were obtained in the presence of $33 \mathrm{mM}$ glucose (Fig. 3C). BPV concentrations of 250 and $300 \mu \mathrm{M}$ were used for one muscle each in the control solution and for three and one muscles, respectively, in the presence of $33 \mathrm{mM}$ glucose. 
BPV concentrations gradually decreased in both the control and insulin-treated groups over 60 minutes. There was a significant interaction effect between the treatment group and time $(P=0.002)$. At each time period from asystole to 60 minutes, the BPV concentrations in the insulin-treated group were slightly lower than those in the control group (Fig. 4).

\section{Non-recirculation experiments - BPV washout}

In all groups, asystole developed at 7-10 minutes following exposure to 500-600 $\mu \mathrm{M} \mathrm{BPV} \mathrm{(Table} \mathrm{1).} \mathrm{A} \mathrm{final}$ concentration of $600 \mu \mathrm{M}$ BPV was used in one and two muscles in the control solution and insulin-treated control solution groups, respectively. After achieving asystole, washout with the control solution induced the first contractile response followed by conduction block, and, after a short time, regular contractions induced by $1.2 \mathrm{~Hz}$ stimulation were restored. The $T_{1 \mathrm{stR}}$ and $\mathrm{T}_{\mathrm{RR}}$ did not differ among the 3 groups (Table 1). Washout with a Tyrode solution for 60 minutes after asystole restored contractility to approximately $60 \%$ of the baseline, while complete recovery was observed after 45 minutes in the insulin- or insulin combined with $33 \mathrm{mM}$ glucose-treated group. Although the changes in contractile forces during recovery were similar between the insulin and insulin combined with $33 \mathrm{mM}$ glucose treatment group and time, there was a significant interaction effect between the control and treatment groups according to time (control vs. insulin, $P=0.013$; control vs. insulin combined with $33 \mathrm{mM}$ glucose, $P=0.001$ ) (Fig. 5).

\section{Discussion}

This study showed that insulin did not reverse BPV-induced conduction block or asystole in isolated cardiac tissues in the recirculation conditions. Although asystole was not reversed, insulin treatment is likely to replenish the energy source that was depleted by high concentrations of BPV.

In the present study, the recirculation condition was used to investigate the effect of insulin on the recovery of stimulated contractile responses, which are dependent on elimination of BPV from tissues. By contrast, the washout condition was used to determine if insulin could enhance or accelerate the timedependent contractile recovery via its metabolic contributions.

In the recirculating condition, neither asystole nor conduction block was reversed by insulin. These findings suggest that insulin does not lower BPV content in cardiac tissues sufficiently to restore $\mathrm{Na}^{+}$ channel function to conduct action potentials. Simply improving metabolic energetics would be ineffective in restoring myocardial contractility when the continuing presence of BPV in the tissue prevents the conduction of action potentials. Considering that myocardial BPV content has a strong positive correlation with aqueous plasma concentration [11], our findings regarding BPV concentrations in aqueous phase indirectly suggest that insulin does not reduce BPV content sufficiently to dissociate BPV from $\mathrm{Na}^{+}$channels in cardiac tissues. It has been reported that insulin had no effect on TTXinhibited $\mathrm{Na}^{+}$channel in rat myoballs [12]. While the exact mechanism behind the gradual decease of $\mathrm{BPV}$ concentration in the control solution remains unclear, this phenomenon may partly be due to ongoing tissue uptake of BPV. 
In most studies using mammalian heart and isolated cardiac muscle preparations, insulin exerted either positive $[6,9,13]$ or no inotropic effect [14]. Whereas our results showed explicit negative inotropy. In this regard, Armin and Bolte [9], in their study using the right ventricular papillary muscles of the guinea pig, demonstrated a dose-response relationship for a positive inotropic effect of insulin. Abe et al. [13] also observed a positive inotropic effect of insulin with the same guinea pig right ventricular papillary muscle preparation. Conversely, our investigations yielded the opposite results despite having used the same species, insulin concentrations, and experimental conditions as the aforementioned studies. Based on the congruency of the animal species and preparation methods, these discrepancies suggest that there was a difference in insulin preparation.

Insulin increases glucose utilization by alterations in membrane kinetics so that glucose transport is accelerated by insulin [15]. With the addition of insulin, glucose uptake increases much more rapidly than increased perfusate concentrations of glucose in the absence of insulin [12]. However, regardless of increased glucose uptake, the positive inotropic effect of insulin has been reported to be independent on the external glucose concentration in the bathing medium $[16,17]$. In the present study, our results demonstrated that insulin had a dose-related negative inotropic effect that also was not affected by external glucose concentration.

However, this may not be applicable for contractile depression induced by BPV application. While insulin concentration-dependently depressed contractility in our result, insulin application following contractile depression by $50 \mu \mathrm{M}$ BPV did not depress contractility further, suggesting certain mechanisms that attenuate further depression by insulin. Of note, in the presence of $33 \mathrm{mM}$ glucose, insulin application increased contractility modestly, but significantly, suggesting that, at least in part, enhanced glucose uptake by insulin is associated with this positive inotropic effect.

With elimination of BPV from the perfusate and its presumed diffusion from the tissue under the washout condition, the contractile responses to $1.2 \mathrm{~Hz}$ stimulation were completely and quickly restored in all muscles as shown in Table 1. This return of contractile responses is consistent with a much faster decrease in myocardial BPV content and restored $\mathrm{Na}^{+}$channel function by washout. In this condition, we observed complete recovery of contractile forces in the insulin- or insulin combined with $33 \mathrm{mM}$ glucosetreated groups while contractility in the control group recovered to approximately $60 \%$ of baseline levels, which indicates long-term injury in tissues by BPV. Of note, differences among the control, insulin, and insulin combined with $33 \mathrm{mM}$ glucose groups for the restoration of contractility appeared to be dependent on whether insulin was used or not. Therefore, the contractile recovery to baseline after treatment with insulin or insulin combined with $33 \mathrm{mM}$ glucose suggests improved myocardial energetics, possibly due to increased cytoplasmic glucose concentrations. These findings imply that although asystole persists under recirculation conditions, metabolic energy is replenished continuously due to enhanced glucose uptake by insulin.

Recently, Kim et al. [5] reported successful resuscitation of BPV-induced circulatory collapse in dogs by insulin. In this study, in association with external chest compression, all insulin/glucose-treated dogs 
were successfully resuscitated, however, none of the control dogs were resuscitated. Based on irreversible restoration of stimulated contractile responses by insulin under recirculation conditions in our results, Kim et al.'s in vivo findings highly suggest the importance of ongoing circulation accomplished with chest compressions to restore cardiac rhythm. Ongoing circulation by chest compression results in gradual redistribution of BPV by increasing blood flow to other organs [5] and possible enhancement of hepatic extraction of BPV [18] would lower BPV concentrations in the myocardium, which contributes to dissociation of BPV from the $\mathrm{Na}^{+}$channel to permit initiation of action potentials. However, a longer period of time was required to reverse impaired myocardial conduction [5]. Considering the redistribution of BPV by chest compression in whole animals or the clinical setting, our results in the recirculation and washout condition from an isolated rodent heart preparation have a limitation to extrapolate to whole animals or humans. However, our results may provide mechanistic insights into the delayed recovery of myocardial conduction and enhanced supply of myocardial energetics which contribute to hemodynamic recovery in whole animals.

Currently, as a mechanism of lipid rescue from BPV-induced cardiac toxicity, two important mechanisms such as lipid sink [19] and metabolic lipid flux effect [1] have been proposed. In the previous studies in in vitro using isolated guinea pig papillary muscles [20] and in an intact rat model of BPV-induced cardiovascular collapse [21], the contractile or hemodynamic recovery has been attributable to lipid sink effect, rather than the metabolic lipid flux effect. Additionally, the liver-targeting property [22] and accelerated clearance of BPV by lipid emulsions $[23,24]$ may add more benefit to decrease the BPV concentrations in cardiac tissues. Although insulin is likely to replenish the depleted ATP, it has no local anesthetic-binding effect, a major disadvantage to be rescued from BPV-induced cardiac toxicity. Based on these aspects, insulin/glucose does not appear to have more benefits than lipid emulsions for the recovery from BPV-induced cardiac arrest.

\section{Conclusions}

Neither insulin nor insulin/glucose treatment does not rescue $\mathrm{Na}+$ channel function to reverse BPVinduced cardiac conduction block or asystole regardless of improved cardiac performance, possibly due to improved myocardial energetics in isolated in vitro animal myocardium model. These findings suggest that treatment with insulin or insulin/glucose is desirable for metabolic energy supply to the myocardium in cases of BPV-induced cardiac collapse. However, considering the importance of decreased BPV concentrations in cardiac tissues, insulin or insulin/glucose cannot achieve satisfactory outcomes.

\section{Abbreviations}

BPV: Bupivacaine; ATP: Adenosine triphosphate; $\mathrm{T}_{\text {asystole: }}$ The time to the appearance of asystole; $\mathrm{T}_{1 \mathrm{stR}}$ : The time to the appearance of the first contractile response; $T_{R R}$ : The time to the first appearance of regular contraction; ANOVA: Analysis of variances; LMM: Linear mixed model; TTX: tetrodotoxin. 


\section{Declarations}

\section{Acknowledgements}

The authors thank Yunho Roh, MS, a Biostatistician in the Biostatistics Collaboration Unit, for statistical analysis and Changhun Park, MS, in the Office for Analytical Support of the Clinical Trial Center in Yonsei University Health System in Seoul, Korea for measurement of bupivacaine concentrations.

\section{Funding}

This research was supported by the Basic Science Research Program through a grant from the National Research Foundation of Korea (NRF) funded by the Ministry of Education, Science and Technology (NRF2012R1A1A2003861). Wyun Kon Park received this fund and contributed substantially to all aspects of this manuscript, including conception and design, acquisition, analysis, interpretation of data, and drafting the article.

\section{Availability of data and materials}

The datasets used or analyzed during the current study are available from the corresponding author on reasonable request.

\section{Authors' contributions}

WKP contributed substantially to all aspects of this manuscript, including conception and design, acquisition, analysis, and interpretation of data and drafting the article. HJK performed the experiments, analyzed the data, and prepared the manuscript. JRJ performed the experiments. CL III interpreted the results of the experiment, analyzed the data, and prepared the manuscript.

\section{Ethics approval and consent to participate}

The experimental procedures were approved by the Yonsei University College of Medicine Animal Research Committee (no. 2013-0406).

\section{Consent for application}

Not applicable.

\section{Competing interests}

The authors declare that they have no competing interests.

\section{Author details}

${ }^{1}$ Department of Anesthesiology and Pain Medicine, and Anesthesia and Pain Research Institute, Yonsei University College of Medicine, Seoul, Korea, ${ }^{2}$ Department of Anesthesiology, University of Virginia Health 
System, Charlottesville, Virginia, USA.

\section{References}

1. Weinberg GL, Palmer JW, VadeBoncouer TR, Zuechner MB, Edelman G, Hoppel CL. Bupivacaine inhibits acylcarnitine exchange in cardiac mitochondria. Anesthesiology. 2000; 92: 523-8.

2. Edelman LB, Ripper R, Kelly K, Di Gregorio G, Weinberg GL. Metabolic context affects hemodynamic response to bupivacaine in the isolated rat heart. Chem Biol Interact. 2008; 172: 48-53.

3. Weinberg G, VadeBoncouer T. Improved energetics may explain the favorable effect of insulin infusion on bupivacaine cardiotoxicity. Anesth Analg. 2001; 92: 1075-6.

4. Kim JT, Jung CW, Lee KH. The effect of insulin on the resuscitation of bupivacaine-induced severe cardiovascular toxicity in dogs. Anesth Analg. 2004; 99: 728-33.

5. Kim MH, Lee KH, Kim CS, Yang S, Uugangerel T, Kim CM, et al. Insulin/glucose infusion successfully resuscitates bupivacaine-induced sudden-onset circulatory collapse in dogs. Can J Anaesth. 2013, 60: 471-8.

6. Stehr SN, Pexa A, Hannack S, Heintz A, Heller AR, Deussen A, et al. Insulin effects on myocardial function and bioenergetics in L-bupivacaine toxicity in the isolated rat heart. Eur $\mathrm{J}$ Anaesthesol. 2007; 24: 340-6.

7. Mazoit JX, Le Guen R, Beloeil H, Benhamou D. Binding of long-lasting local anesthetics to lipid emulsions. Anesthesiology. 2009; 110: 380-6.

8. Lynch C III. Differential depression of myocardial contractility by halothane and isoflurane in vitro. Anesthesiology. 1986; 64:620-31.

9. Armin TV, Bolte HD. Dose-response relationship for a positive inotropic effect of insulin on isolated papillary muscle. Klin Wochenschr. 1980; 58: 537-9.

10. Weisenfeld SW, Podolsky S, Goldsmith L, Ziff L. Adsorption of insulin to infusion bottles and tubing. Diabetes. 1968; 17: 766-71.

11. Weinberg G, Lin B, Zheng S, Di Gregorio G, Hiller D, Ripper R, et al. Partitioning effect in lipid resuscitation: further evidence for the lipid sink. Crit Care Med. 2010; 38: 2268-9.

12. Zierler $\mathrm{K}, \mathrm{Wu} \mathrm{FS}$. An early outward transient $\mathrm{K}^{+}$current that depends on a preceding $\mathrm{Na}^{+}$current and is enhanced by insulin. Pflügers Arch. 1992; 22: 267-72.

13. Abe K, Oka M, Kubo K, Takenoshita Y, Frey R. Response of isolated guinea pig myocardium to insulin therapy during normothermia and graded hypothermia. Resuscitation 1986; 13:107-13.

14. Markovitz LJ, Hasin Y, Freund HR. The effect of insulin and glucagon on systolic properties of the normal and septic isolated rat heart. Basic Res Cardiol 1985; 80:377-83.

15. Morgan HE, Henderson MJ, Regen DM, Park CR. Regulation of glucose uptake in muscle. I. The effects of insulin and anoxia on glucose transport and phosphorylation in the isolated, perfused heart of normal rats. J Biol Chem. 1961;236: 253-61. 
16. Lucchesi BR, Medina M, Kniffen FJ. The positive inotropic action of insulin in the canine heart. Eur J Pharmacol. 1972;18: 107-15.

17. Lee JC, Downing SE. Effects of insulin on cardiac muscle contraction and responsiveness to norepinephrine. Am J Physiol. 1976;230: 1360-5.

18. Thomas JM, Schug SA. Recent advances in the pharmacokinetics of local anesthetics. Long-acting amide enantiomers and continuous infusions. Clin Pharmacokinet. 1999;36: 67-83.

19. Weinberg GL, VadeBoncouer T, Ramaraju GA, Garcia-Amaro MF, Cwik MJ. Pretreatment or resuscitation with a lipid infusion shifts the dose-response to bupivacaine-induced asystole in rats. Anesthesiology. 1998;88: 1071-75.

20. Park WK, Kim HS, Kim SW, Jung JR, Lynch C III, Min NH. Intralipid restoration of myocardial contractions following bupivacaine-induced asystole: concentration- and time-dependence in vitro. Anesth Analg. 2017; 125: 91-100.

21. Li Z, Xia Y, Dong X, Chen H, Xia F, Wang X, et al. Lipid resuscitation of bupivacaine toxicity: long-chain triglyceride emulsion provides benefits over long- and medium-chain triglyceride emulsion. Anesthesiology. 2011; 115: 1219-28.

22. Jang JH, Kim CK, Choi HG, Sung JH. Preparation and evaluation of 2-(allythio)pyrazine-loaded lipid emulsion with enhanced stability and liver targeting. Drug Dev Ind Pharm. 2009; 35: 363-8.

23. Shi K, Xia Y, Wang Q, Wu Y, Dong X, Chen C, et al. The effect of lipid emulsion on pharmacokinetics and tissue distribution of bupivacaine in rats. Anesth Analg. 2013;116: 804-9.

24. Tang W, Wang Q, Shi K, Dong J, Lin S, Zhao S, et al. The effect of lipid emulsion on pharmacokinetics of bupivacaine in rats: long-chain triglyceride versus long- and medium-chain triglyceride. Anesth Analg. 2016;123: 1116-22.

\section{Tables}

Table 1 Times to appearance of $\mathrm{T}_{\text {asystole }}, \mathrm{T}_{1 \mathrm{stR}}$, and $\mathrm{T}_{\mathrm{RR}}$ in each group following application of 500-600 $\mu \mathrm{M}$ bupivacaine under non-recirculation conditions

\begin{tabular}{|l|c|c|c|c|}
\hline & $\mathrm{N}$ & $\begin{array}{c}\mathrm{T}_{\text {asystole }} \\
(\mathrm{min})\end{array}$ & $\begin{array}{c}\mathrm{T}_{1 \mathrm{stR}} \\
(\mathrm{min})\end{array}$ & $\begin{array}{c}\mathrm{T}_{\mathrm{RR}} \\
(\mathrm{min})\end{array}$ \\
\hline Control & 7 & $10.73 \pm 6.86$ & $5.62 \pm 2.61$ & $12.14 \pm 6.0$ \\
\hline Insulin & 9 & $7.49 \pm 4.61$ & $5.96 \pm 2.94$ & $11.28 \pm 4.85$ \\
\hline Insulin/glucose & 11 & $9.28 \pm 5.46$ & $4.70 \pm 2.86$ & $10.02 \pm 2.99$ \\
\hline
\end{tabular}

Results are presented as mean \pm SD. There were no significant differences among the four groups with regard to $\mathrm{T}_{\text {asystole, }} \mathrm{T}_{1 \mathrm{stR}}$, or $\mathrm{T}_{\mathrm{RR}} \cdot \mathrm{N}=$ number of papillary muscles. Min $=$ 
minutes. $\mathrm{T}_{\text {asystole }}=$ time to appearance of asystole. $\mathrm{T}_{1 \mathrm{stR}}=$ time to appearance of the first contractile response. $\mathrm{T}_{\mathrm{RR}}=$ time to appearance of the regular contractile response. Insulin/glucose indicates insulin application in the presence of $33 \mathrm{mM}$ glucose.

\section{Figures}

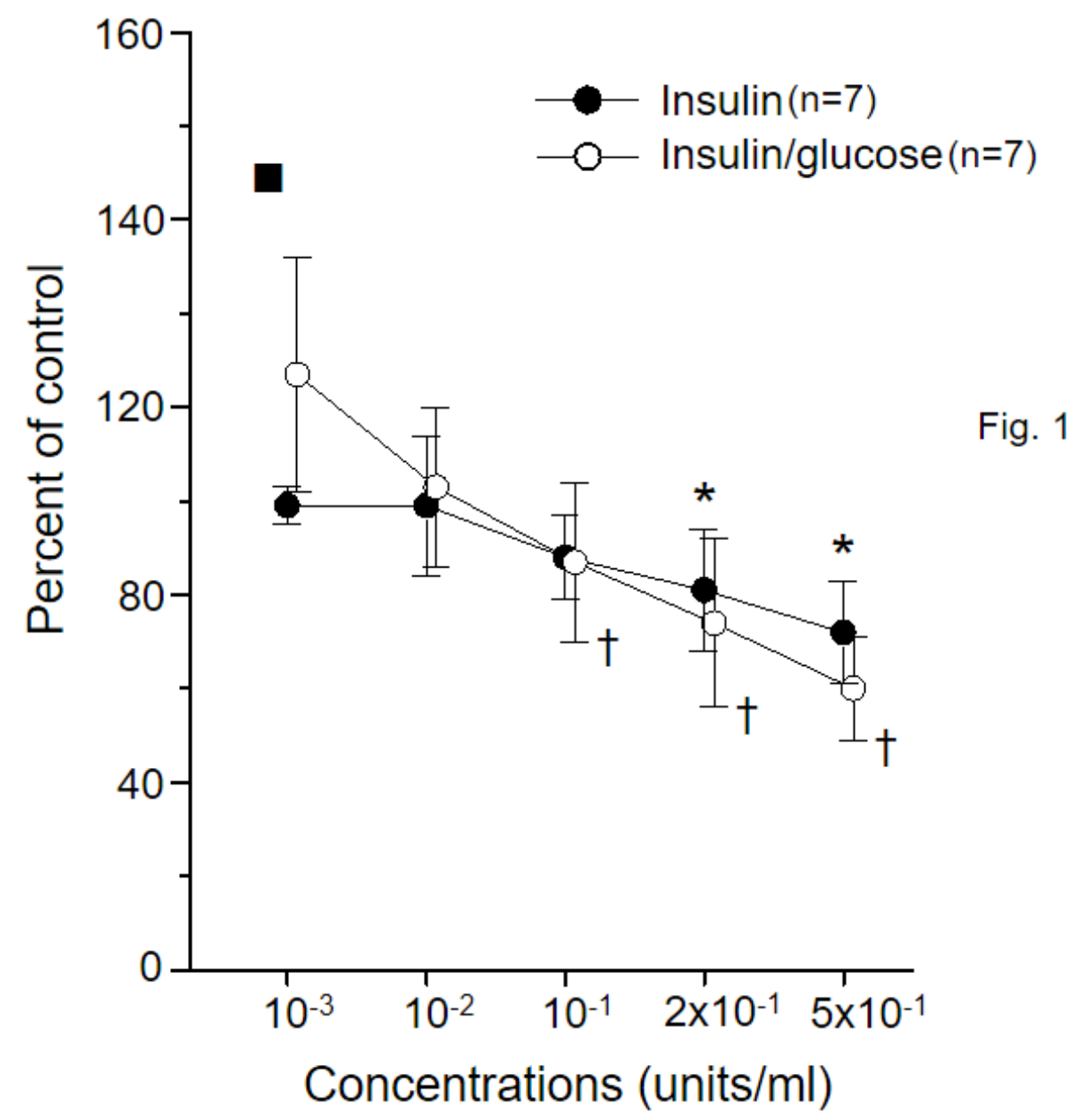

Figure 1

Effects of different concentrations of insulin on contractile forces (dF/dt-max) in the presence of normal $(11 \mathrm{mM})(\square)$ and $33 \mathrm{mM}$ glucose (insulin/glucose) () under recirculation conditions. The black square $(\square)$ in the left upper corner indicates the percent of the control $\mathrm{dF} / \mathrm{dt}$-max following exposure to $33 \mathrm{mM}$ glucose before application of insulin. * (insulin) or $\dagger$ (insulin/glucose) indicates $P<0.05$ vs. baseline obtained with plain Tyrode solution at $1.2 \mathrm{~Hz}$ stimulation rate. $P$ values are as follows. () $2 \times 10-1$ unit/ml vs. baseline, $P=0.001 ; 5 \times 10-1$ unit/ml vs. baseline, $P<0.001$. () $10-1,2 \times 10-1$, and $5 \times 10-1$ 
units $/ \mathrm{ml}$ vs. baseline, $\mathrm{P}<0.05$, respectively. The baseline $\mathrm{dF} / \mathrm{dt}$-max values were $4.71 \pm 2.08$ and $7.78 \pm$ $3.12 \mathrm{mN} / \mathrm{sec}$ in the insulin or insulin/glucose group, respectively.

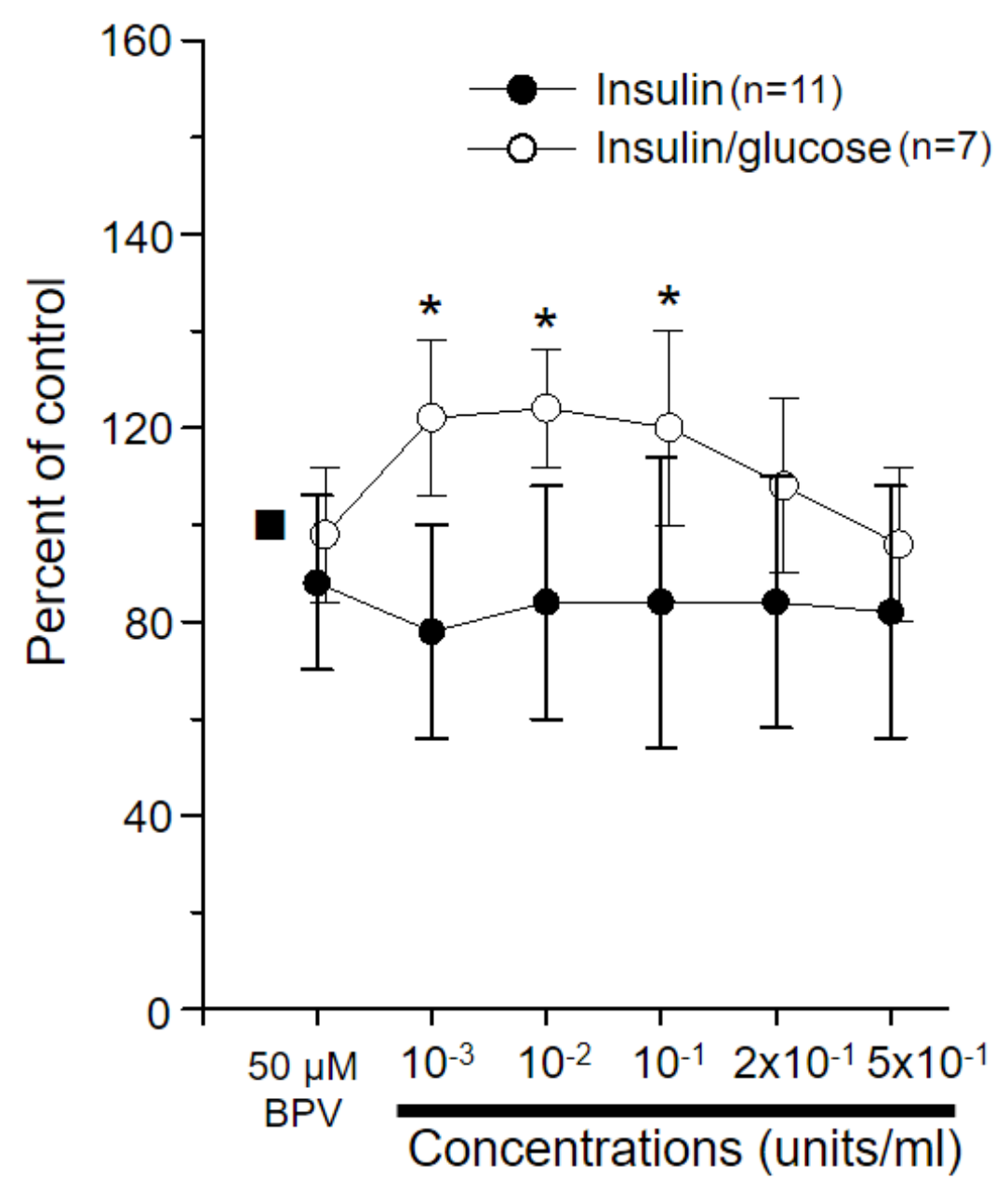

Fig. 2

\section{Figure 2}

Effects of various concentrations of insulin on contractile forces following application of $50 \mu \mathrm{M}$ bupivacaine (BPV) in the presence of normal (11 mM) and () $33 \mathrm{mM}$ glucose (insulin/glucose) () under recirculation conditions. The black square $(\square)$ in the left upper corner indicates the percent of the control $\mathrm{dF} / \mathrm{dt}$-max following exposure to $33 \mathrm{mM}$ glucose under recirculation conditions. * (insulin/glucose) indicates $\mathrm{P}<0.05 \mathrm{vs}$. baseline obtained with plain Tyrode solution at $1.2 \mathrm{~Hz}$ stimulation rate. $\mathrm{P}$ values are as follows. ( $($ ) $50 \mu \mathrm{M}$ BPV, 10-3, 10-2, 10-1, $2 \times 10-1$, and $5 \times 10-1$ units/ml of insulin vs. control, $\mathrm{P}<0.001$. $10-3,10-2,10-1,2 \times 10-1$, and $5 \times 10-1$ units/ml of insulin vs. $50 \mu \mathrm{M} B P V, P>0.05$. (ه) $50 \mu \mathrm{M} \mathrm{BPV}, 10-3,10-$ $2,10-1,2 \times 10-1$, and $5 \times 10-1$ units $/ \mathrm{ml}$ of insulin vs. control, $\mathrm{P}<0.001 .10-3 \mathrm{unit} / \mathrm{ml}$ vs. $50 \mu \mathrm{M} \mathrm{BPV}, \mathrm{P}=$ $0.012 ; 10-2$ unit/ml vs. $50 \mu \mathrm{M} \mathrm{BPV}, \mathrm{P}=0.006 ; 10-1 \mathrm{unit} / \mathrm{ml}$ vs. $50 \mu \mathrm{M} \mathrm{BPV}, \mathrm{P}=0.010 ; 2 \times 10-1 \mathrm{unit} / \mathrm{ml}$ vs. $50 \mu \mathrm{M}$ BPV, $\mathrm{P}=0.115 ; 5 \times 10-1$ unit $/ \mathrm{ml}$ vs. $50 \mu \mathrm{M}$ BPV, $\mathrm{P}=0.748 ; 2 \times 10-1$ unit $/ \mathrm{ml}$ vs. $5 \times 10-1, \mathrm{P}=0.115 ; 5$ $x$ 10-1 vs. 10-3 unit $/ \mathrm{ml}, P=0.007$. The baseline $\mathrm{dF} / \mathrm{dt}$-max values were $4.97 \pm 3.01$ and $2.16 \pm 0.69$ $\mathrm{mN} / \mathrm{sec}$ in the insulin or insulin/glucose group, respectively. 


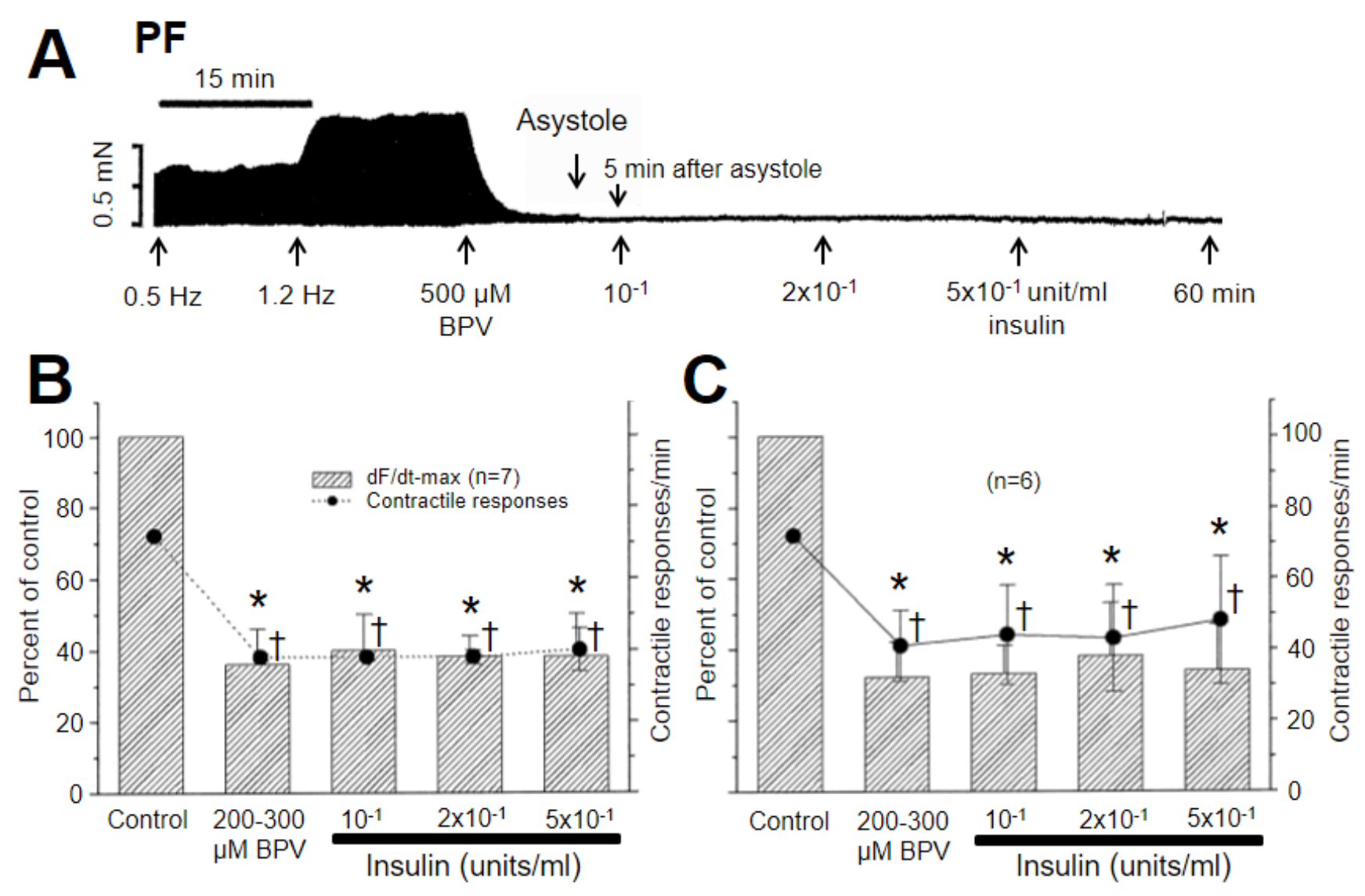

Fig. 3

\section{Figure 3}

A. Effects of various concentrations of insulin on bupivacaine (BPV)-induced asystole under recirculation condition. $\mathrm{B}$ and $\mathrm{C}$. Effects of various concentrations of insulin on stimulated contractile responses and contractile forces (dF/dt-max) following application of 200-300 $\mu \mathrm{M}$ BPV in the presence of a normal glucose concentration (11 mM) (B) and $33 \mathrm{mM}$ glucose (C) under recirculation conditions. Min = minutes. (B) * or + : $P<0.001$ vs. control. Between $200 \mu \mathrm{M}$ BPV and each concentration of insulin, $P>0.05$. (C) * or t: $P<0.001$ vs. control. Between 200-300 $\mu \mathrm{M}$ BPV and each concentration of insulin, $P>0.05$. ${ }^{*}$ and $\dagger$ in $\mathrm{B}$ and $\mathrm{C}$ indicate contractile forces and stimulated contractile responses, respectively. The baseline $\mathrm{dF} / \mathrm{dt}-$ max values were $3.53 \pm 0.89$ and $5.09 \pm 2.13 \mathrm{mN} / \mathrm{sec}$ in the insulin or insulin combined with $33 \mathrm{mM}$ glucose group, respectively. 


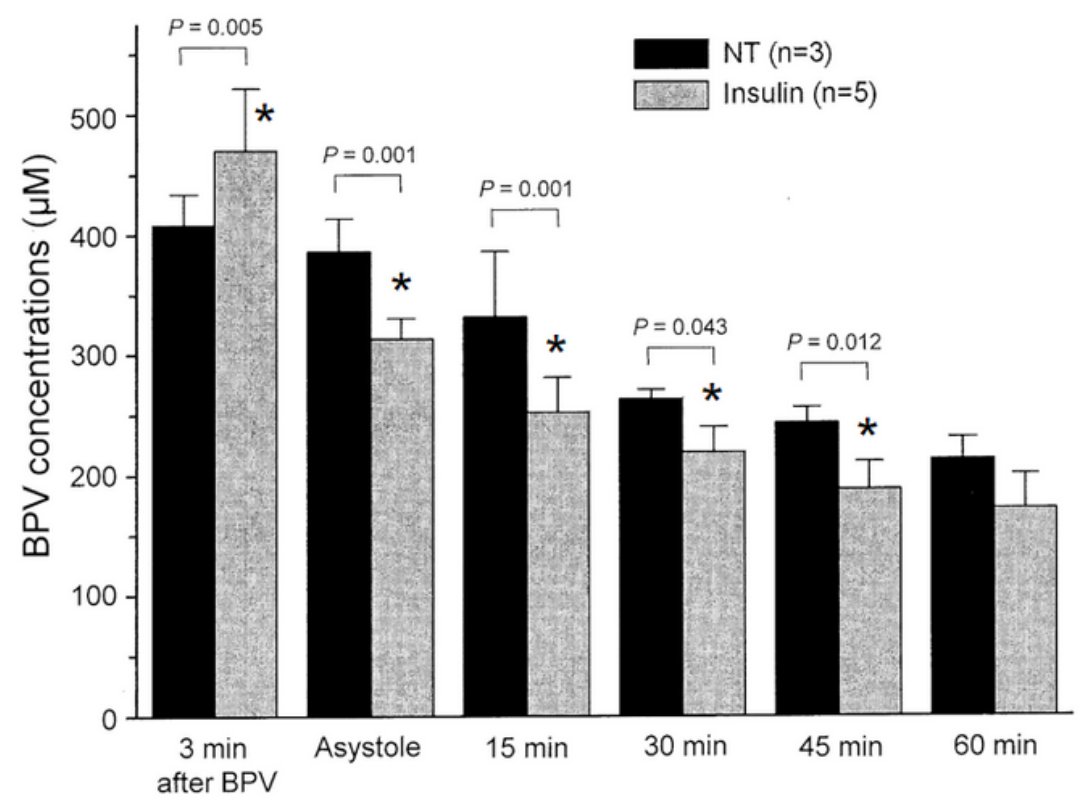

Fig. 4

\section{Figure 4}

Changes in bupivacaine (BPV) concentrations following asystole induced by $500 \mu \mathrm{M}$ BPV for 60 minutes at each time period in modified normal Tyrode solution (NT) and $2 \times 10-1 \mathrm{unit} / \mathrm{ml}$ insulin-treated NT solution under recirculation conditions. * indicates $\mathrm{P}$ value $<0.05$ at each time point between the 2 groups. 


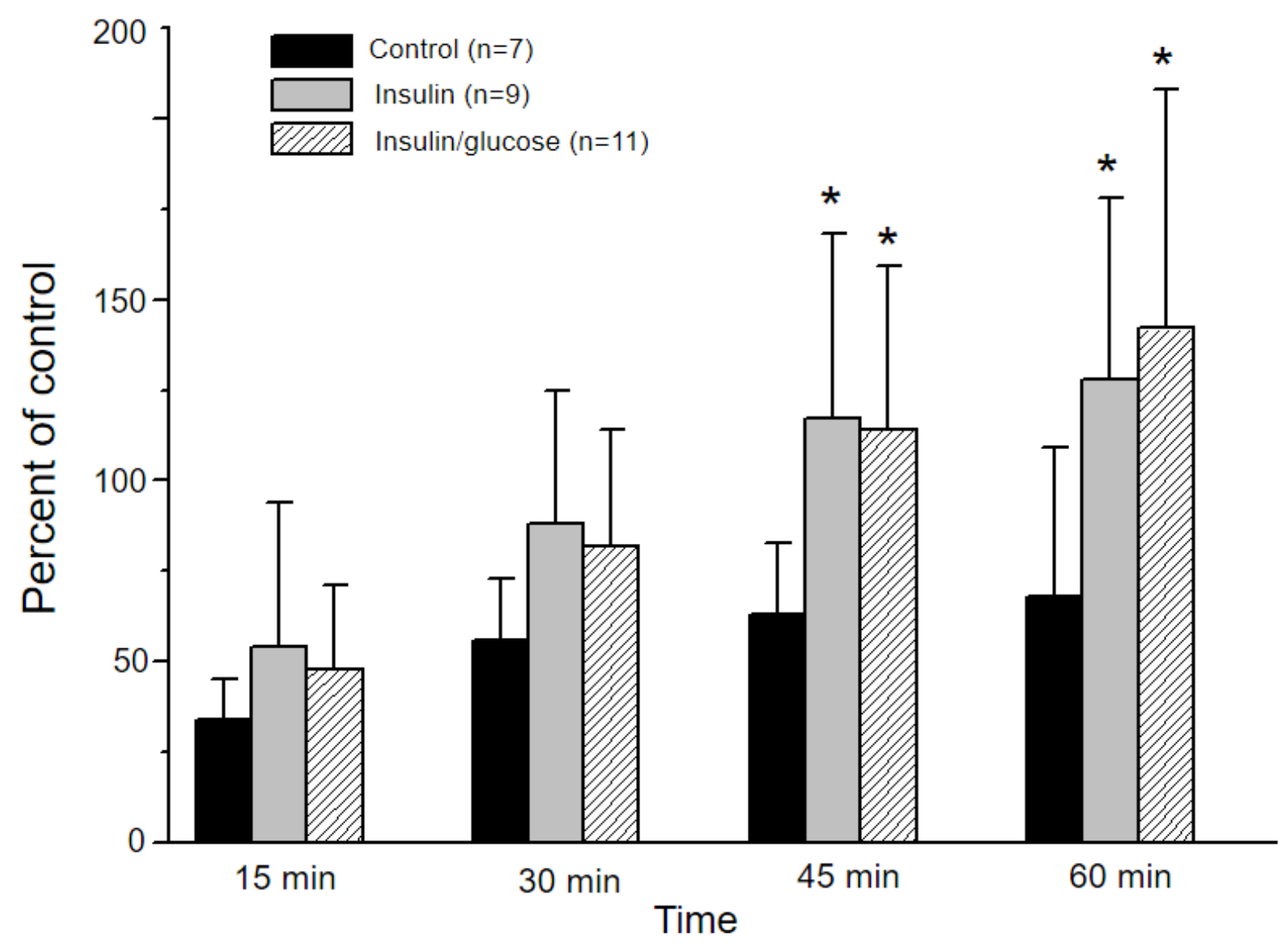

Fig. 5

\section{Figure 5}

Recovery of contractile forces (dF/dt-max) for 60 minutes after a wash with control (modified normal Tyrode) solution, control solution containing $2 \times 10-1$ unit/ml of insulin (insulin), and control solution containing $33 \mathrm{mM}$ glucose and $2 \times 10-1 \mathrm{unit} / \mathrm{ml}$ of insulin (insulin/glucose) following asystole induced by $500 \mu \mathrm{M}$ bupivacaine under non-recirculation conditions. Min = minutes. * indicates $\mathrm{P}$ value $<0.05$ at each time point compared to control group. $P$ values at each time period among the 3 groups are as follows. 45 minutes: control vs. insulin, $P=0.0276$; control vs. insulin/glucose, $P=0.0031$; insulin vs. insulin/glucose, $P=0.3931 .60$ minute: control vs. insulin, $P=0.0214$; control vs. insulin/glucose, $P=$ 0.001 ; insulin vs. insulin/glucose, $\mathrm{P}=0.2387$. The baseline $\mathrm{dF} / \mathrm{dt}$-max values were $4.41 \pm 3.25,4.16 \pm$ 2.46 , and $3.70 \pm 2.07 \mathrm{mN} / \mathrm{sec}$ in the groups treated with control solution, insulin, and insulin/glucose, respectively.

\section{Supplementary Files}

This is a list of supplementary files associated with this preprint. Click to download. 
- NC3RsARRIVEGuidelinesChecklist2014WordFormatWKP.docx 